\title{
Pulse oximetry reference values at high altitude
}

\author{
Juan Manuel Lozano, Olga Rebeca Duque, Tatiana Buitrago, Soad Behaine
}

\begin{abstract}
Pulse oximetry is becoming popular for measuring oxygen saturation of haemoglobin in paediatric patients. There are no reference values for children living at high altitudes, and the aim of this study was to determine the values of oxygen saturation of haemoglobin in healthy children in Bogota ( $2640 \mathrm{~m}$ above sea level).

The saturation was determined in 189 children aged 5 days to 24 months with a Nellcor N10 oximeter. Mean values and 95\% confidence intervals (CI) were calculated. Analysis of variance was used for assessing the differences in saturation with age and physiological status. The values were normally distributed with a mean (SD) of $93.3(2.05) \%$ and $95 \% \mathrm{CI}$ of $93.0 \%$ to $93.6 \%$. There were no clinically important differences in the oxygen saturation of haemoglobin in the range of ages included. The mean saturation in sleeping children was significantly lower than that during other physiological states $(91 \cdot 1 \% v$ 93.3\%) but the difference was not clinically important.
\end{abstract}

As expected, the values for the oxygen saturation of haemoglobin in the children studied were lower than those reported from children living at sea level. These estimates can be used for interpreting results of the oxygen saturation in haemoglobin in children from Bogota and other cities located at a similar altitude.

Pulse oximetry is a method for continuous monitoring of the oxygen saturation of haemoglobin. The studies of validation of this method performed to date in paediatric patients have compared the results of the oxygen saturation of haemoglobin with the oxyhemoglobin saturation of arterial blood $\left(\mathrm{SaO}_{2}\right)$ and with the transcutaneous pressure of oxygen. These reports have shown correlation coefficient values between 0.86 and 0.99 , and mean differences between oxygen saturation of haemoglobin and $\mathrm{SaO}_{2}$ in the range from $+0 \cdot 2 \%$ to $+2 \cdot 9 \% .^{1-7}$ All these studies confirm a strong correlation between the oxygen saturation of haemoglobin and other techniques of measuring the $\mathrm{SaO}_{2}$. One additional study, which included subjects with severe hypoxaemia, suggests that pulse oximetry can be less precise when oxygen saturation of haemoglobin is below $65 \% .^{8}$ Pulse oximetry has been reliable for monitoring purposes in subjects of all ages during anaesthesia and in intensive care units, providing accurate data. ${ }^{19} 10$
To our knowledge there are no previous reports of reference values for this technique in children living at high altitude. The goal of this study was to determine the values of the oxygen saturation of haemoglobin measured by pulse oximetry in a sample of normal children living in the city of Bogota ( $2640 \mathrm{~m}$ above sea level).

\section{Subjects and methods}

A descriptive observational study was conducted. During the months of January to March of 1990 we studied a group of 190 clinically healthy children aged 5 days to 24 months inclusive, born and permanently residing in the city of Bogota. The subjects were selected from the outpatient departments of the San Ignacio's University Hospital and the Colsubsidio Children's Hospital. These two hospitals are tertiary referral centres that also provide primary care for ambulatory patients. Children were excluded from the study because of: (1) any respiratory symptom in the two weeks before the examination. (2) Any abnormal sign on respiratory and/or cardiovascular clinical examination. (3) A history of chronical pulmonary or cardiovascular disease. (4) A history of neonatal respiratory illness. (5) Blood transfusions during the previous six months. (6) Malnutrition (height to weight relation below fifth centile on the National Center for Health Statistics tables). One of the subjects was later excluded because after reviewing his data the relation of height to weight was below that required for participation. Table 1 summarises the main demographic characteristics of the children studied. The study protocol was approved by the research committees of both health centres. Informed consent was obtained from parents before including any patient.

All measurements were done with a Nellcor N10 oximeter by one of the researchers (ORD). To determine the oxygen saturation of haemoglobin an adhesive sensor was placed on the

Table 1 Distribution of subjects according to age and sex

\begin{tabular}{ll}
\hline & $N o(\%)$ \\
\hline Age (months): & \multicolumn{1}{c}{ No } \\
\hline $1-3$ & $39(20 \cdot 6)$ \\
$1-6$ & $35(18 \cdot 5)$ \\
$4-6$ & $24(12 \cdot 7)$ \\
$7-12$ & $23(12 \cdot 2)$ \\
$13-18$ & $38(20 \cdot 1)$ \\
$19-24$ & $30(15 \cdot 9)$ \\
Total & $189(100)$ \\
Sex: & \\
$\quad$ Male & $98(51 \cdot 9)$ \\
Female & $91(48 \cdot 1)$ \\
\hline
\end{tabular}


index finger on the hand of the subject. The oximeter was set on normal mode. A measurement was considered successful when the oximeter displayed the subject's cardiac rate, which requires a reading by the machine of at least 10 seconds. The oximeter pulse amplitude indicator displayed on the screen was compared with the simultaneous measurement of the radial pulse obtained by palpation in the other hand to be sure that signals were coincident. If the sensor was not able to track the pulse during the measurement period due to excessive patient motion, no saturation reading was obtained and a check sensor signal was displayed. In these cases the measurement was repeated until the oxygen saturation of haemoglobin was obtained. All readings were duplicated and were taken if possible with the child awake and resting. When this was not possible, measurement conditions (crying, sleeping, or feeding) were noted. When exposed to a source of bright light (for example during phototherapy) the sensor was covered or the source of light was temporarily turned off. In order to demonstrate that the pulse oximeter was functioning properly, simultaneous measurements of oxygen saturation of haemoglobin and $\mathrm{SaO}_{2}$ were performed in a group of 11 patients admitted to the intensive care unit. Blood samples obtained by arterial puncture were processed on a Hemoximeter OSM2 co-oximeter (Radiometer). The concordance between the oxygen saturation of haemoglobin and $\mathrm{SaO}_{2}$ was very high, with an intraclass correlation coefficient of 0.98 .

As the data had a normal distribution, means and $95 \%$ confidence intervals (CI) were calculated for all age and clinical status groups and compared by analysis of variance. The concordance between our oximeter and arterial blood saturation readings was assessed calculating the intraclass correlation coefficient. ${ }^{11}$

\section{Results}

As the data for the oxygen saturation of haemoglobin were distributed on an unimodal and symmetric fashion, mean (SD) values were calculated. The lowest and highest values were $84 \%$ and $100 \%$, respectively, with a mean (SD) of $93.3(2 \cdot 05) \%($ median $=93 \cdot 0$, mode $=93 \cdot 0)$. The $95 \% \mathrm{CI}$ for the mean oxygen saturation of haemoglobin was $93.0 \%$ to $93 \cdot 6 \%$. Mean values and $95 \% \mathrm{CI}$ for the different age groups were also calculated and are summarised in table 2 . The mean value for oxygen saturation of haemoglobin in children aged less than 1 month was statistically different from that in the group

Table 2 Mean (\%) value for the oxygen saturation of haemoglobin in different age groups

\begin{tabular}{lll}
\hline Age (months) & Mean (\%) & $95 \%$ CI \\
\hline$<1$ & 92.6 & $92 \cdot 1$ to $93 \cdot 2^{*}$ \\
$1-3$ & 93.5 & 92.6 to $94 \cdot 4$ \\
$4-6$ & 93.5 & 92.6 to $94 \cdot 4$ \\
$7-12$ & 93.4 & 92.4 to $94 \cdot 4$ \\
$13-18$ & 93.7 & 93.2 to $94 \cdot 3^{*}$ \\
$19-24$ & 93.0 & 92.3 to 93.7 \\
Total & 93.3 & 93.0 to 93.6 \\
\hline
\end{tabular}

* $<1$ month $v 13-18$ months, $\mathrm{p}<0.05$ by analysis of variance.
Table 3 Mean (\%) value for the oxygen saturation of haemoglobin in different physiological states

\begin{tabular}{lcl}
\hline State & $\begin{array}{l}\text { No of } \\
\text { infants }\end{array}$ & $\begin{array}{l}\text { Mean } \\
(\%)\end{array}$ \\
\hline Quiet wakefulness & 150 & $93 \cdot 4$ \\
Sleeping & 8 & $91 \cdot 1^{*}$ \\
Crying & 15 & $93 \cdot 3$ \\
Sucking & 16 & $93 \cdot 5$ \\
Total & 189 & $93 \cdot 3$ \\
\hline
\end{tabular}

${ }^{*} \mathrm{p}<0 \cdot 05$ by analysis of variance.

who were between 13 and 18 months of age (92.6\% and $93.7 \%$, respectively; $\mathrm{p}<0.05$, one way analysis of variance)

Table 3 presents the distribution depending on the physiological state of the child when conducting the measurements, as well as the mean value for oxygen saturation of haemoglobin in each group. Most of the readings were done with the subject alert and resting. A few measurements were obtained with the child crying, sleeping, or sucking. The mean value of the oxygen saturation of haemoglobin in the group of children sleeping was slightly lower (91.1\% and between $93.3 \%$ and $93.5 \%$ for sleeping and other states, respectively). This difference was statistically significant $(p<0.05$, one way anaysis of variance).

\section{Discussion}

Up to the present two published studies have evaluated the values of the oxygen saturation of haemoglobin in both preterm and term healthy newborns, as well as in infants up to 6 months of age. ${ }^{12}{ }^{13}$ These two studies were conducted in the Hospital for Sick Children in Toronto, Canada, a city that is less than $100 \mathrm{~m}$ above sea level. As individuals living at high altitude are in a hypobaric state, their normal oxygen tension and $\mathrm{SaO}_{2}$ values may be different to those from individuals who live at lower altitudes. ${ }^{14}$ To date, there have been no reports of reference values for oxygen saturation in haemoglobin in the paediatric population living at high altitude. These reference values will be helpful both in clinical practice and in research. As was expected by the lower barometric pressure of Bogota, the mean value for the oxygen saturation of haemoglobin obtained in our study is below that reported in healthy children in Toronto $(93.3 \%$ and $96.4 \%$ respectively). ${ }^{12}$ The lowest value observed in our sample was $84 \%$ and was obtained from a sleeping 2 month old infant. The highest value was $100 \%$ observed in a 10 month old crying child. It is not likely that these extreme readings were due to an oximeter dysfunction, because these data were confirmed on three different readings. These extreme values are uncommon in healthy children residing in Bogota.

As the main goal of our study was to obtain the values for oxygen saturation of haemoglobin on healthy children, it was necessary to select the study subjects carefully. The criteria used to select the sample were rigorous. None of the children included suffered from serious diseases, particularly acute or chronic cardiopulmonary illnesses. The data presented in table 1 show 
that an adequate representation of both sexes and of all ages in the two first years of life was achieved. The $95 \% \mathrm{CI}$ for the mean values obtained were $93.0 \%$ to $93.6 \%$.

The mean value for the oxygen saturation of haemoglobin observed in children aged less than 1 month $(92 \cdot 6 \%)$ was statistically different from that in children between 13 and 18 months of age $(93.7 \%)$. Whether this small difference is clinically important remains to be clarified. The reason for the observed difference is not clear. Although we did not measure the proportion of fetal haemoglobin in any of the study subjects, the higher content expected in younger infants does not explain it, because the deviation to the left of the fetal haemoglobin saturation curve may produce higher oxygen saturation readings for fetal haemoglobin than for adult haemoglobin for a given $\mathrm{PaO}_{2},{ }^{6}$ and the difference observed by us was the opposite. Most authors agree that pulse oximetry provides reliable results even in presence of high concentrations of fetal haemoglobin. ${ }^{1} 3$ 15 16

The studies conducted by Mok et al demonstrated that the normal oxygen saturation of haemoglobin can be lower during feeding and active sleep when compared with the results obtained in the same children during rest or quiet sleep. ${ }^{12} 13$ For this reason we tried to obtain the saturation values with the children at rest. This was possible for the great majority of our subjects $(79 \cdot 4 \%)$. Even though it was not the main goal of the study, our data also suggest that the readings obtained during sleep are lower than during other physiological states. However, these results must be interpreted with caution, because the number of individuals sleeping during the measurement was small (eight), and the very low saturation observed in one of them $(84 \%)$ would affect the mean value for this group. When the analysis was repeated without this very low reading, the mean oxygen saturation of haemoglobin in the group of children sleeping was lower than that observed in the other physiological states $(92 \cdot 1 \%)$, but was no longer significant. The practical effects of the various values found among children sleeping and awake $(91 \cdot 2 \%$ and between $93.3 \%$ and $93 \cdot 5 \%$, respectively) are probably small.

The validity of readings of the oxygen saturation of haemoglobin obtained in the 15 crying children deserves further discussion because of the possibility of error due to movement artifacts. ${ }^{17}$ Several measurements were repeated because of signals indicating movement before obtaining the saturation figures. Obtaining saturation values with the N10 oximeter means that there is no interference with the measurement (even if the subject is crying). We included those values in order to make the results applicable to children who cry during the measurement. Although only $7 \cdot 9 \%$ of our subjects were crying, the proportion of 'unsuccessful measurements' due to crying, restlessness, fear of the probe, lack of cooperation, and movement reported by other authors may be as high as $13 \%$ and $9 \%$ for infants aged less than 10 months between 1 and 6 years, respectively. ${ }^{18}$

\section{CONCLUSIONS}

We believe that the population of our study was representative of the population of healthy children of the city of Bogota and of other Latin American cities located at similar altitudes. We hope that the results of our study can be used with additional reference values for the interpretation of the readings for oxygen saturation of haemoglobin at high altitude.

The study was partially supported by the Center for Immunization Research, The Johns Hopkins University, and by the Rockefeller Foundation.

1 Durand M, Ramanathan R. Pulse oximetry for continuous monitoring in sick newborn infants. $\mathcal{F}$ Pediatr $1986 ; 109$ : $1052-6$.

2 Fanconi S, Doherty P, Edmonds J, Barker G. Bohn D. Pulse oximetry in pediatric intensive care: comparison with measured saturations and transcutaneous oxygen tension. f Pediatr 1985;107:362-6.

3 Ramanathan R, Durand M, Larrazabal C. Pulse oximetry in very low birth weight infants with acute and chronic lung disease. Pediatrics 1987;79:612-7.

4 Deckardt R, Steward DJ. Noninvasive arterial hemoglobin oxygen saturation vs transcutaneous oxygen tension monitoring in the preterm infant. Crit Care Med 1984;12 935-9.

5 Hay WW, Brockway JM, Eyzaguirre M. Neonatal pulse oximetry: accuracy and reliability. Pediatrics 1989;83: 717-22.

6 Jennis MS, Peabody J. Pulses oximetry: an alternative method for the assessment of oxygenation in newborn method for the assessment of oxy
infants. Pediatrics 1987;79:524-8.

7 Southall DP, Bignall S, Stebbens VA, Alexander JR, Rivers RPA, Lissauer T. Pulse oximeter and transcutaneous arterial oxygen measurements in neonatal and paediatric intensive care. Arch Dis Child 1987;62:882-8.

8 Fanconi S. Reliability of pulse oximetry in hypoxic infants. 7 Pediatr 1988;112:424-7.

9 Barker SJ, Tremper KK. Pulse oximetry: applications an limitations. Int Anesthesiol Clin 1987;25:155-75.

10 Bowes WA, Cokke BC, Hulka J. Pulse oximetry: a review of the theory, accuracy and clinical complications. Obstet Gynecol 1989;74:541-6.

11 Kramer MS, Feinstein AR. Clinical biostatistics. LIV. The statistics of concordance. Clin Pharmacol Ther 1981;29. $111-22$

12 Mok J, Hak H, McLaughlin FJ, Pinter M, Canny G, Levison $H$. Effects of age and state of wakefulness on transcutaneous oxygen values in preterm infants: a longitudinal study. oxygen values in preterm

13 Mok J, McLaughlin FJ, Pintar M, Hak H, Amaro-Galves R Levison $\mathrm{H}$. Transcutaneous monitoring of oxygenation: Levison H. Transcutaneous monitoring of

14 Lenfant $\mathrm{C}$, Silluivan $\mathrm{K}$. Adaptation to high altitude. $\mathrm{N} \mathrm{Englf}$ Med 1981;284:1298.

15 Hay WW. The uses, benefits and limitations of pulse oximetry in neonatal medicine: consensus on key issues. F Perinatol 1987;7:347-9.

16 Harris A, Sendak MJ, Donham RT, Thomas M, Duncan D. Absorption characteristics of human fetal hemoglobin a wavelengths used in pulse oximetry. $\mathcal{F}$ Clin Monit 1988;4 175-7.

17 Huch A, Huch R, Konig V, et al. Limitations of pulse oximetry. Lancet 1988;i:357-8.

18 Levene S, McKenzie SA. Pulse oximetry in children. Lancet 1988;i:415-6. 